\title{
Editorial
}

\section{Proveer, cuidar y criar: evidencias, discursos y experiencias sobre paternidad en América Latina}

\author{
Florencia Herrera \\ Universidad Diego Portales, Santiago, Chile \\ Email: florencia.herrera@udp.cl \\ Francisco Aguayo \\ Universidad Católica de Valparaíso, Valparaiso, Chile. \\ Email: francisco.aguayo.f@mail.pucv.cl \\ Jael Goldsmith Weil \\ Universidad Central de Chile, Santiago, Chile \\ Email: jael.goldsmith@ucentral.cl
}

La participación de los padres hombres en el cuidado, la crianza y las tareas domésticas es un asunto clave para la igualdad de género y el desarrollo infantil. En Latinoamérica las mujeres destinan más tiempo que los hombres al cuidado en el hogar(Rico y Robles, 2016). Por otra parte las políticas han reforzado el papel de cuidadoras en las madres y de proveedores económicos en los padres. En las últimas décadas las mujeres salieron a trabajar remuneradamente sin embargo todavía dedican más tiempo al cuidado y sus trabajos son más precarios (Blofield y Martínez, 2014). A pesar de todos los avances en legislación y políticas, al parecer, el orden de género se resiste a cambiar o lo hace muy lentamente.

Si bien, hay una producción académica considerable sobre los desafíos que presentan los cambios demográficos y del mercado de trabajo y la distribución de tareas de cuidado entre estado, mercado y mujeres-madres; la discusión regional enfocada en los padres es aún incipiente y ha sobrerrepresentado al padre heterosexual de clase media que forma parte de una familia biparental.El concepto de maternalismo, entendido como la ideología, retórica, prácticas y políticas públicas que amalgaman mujeres y madres ha ganado tracción en discusiones académicas recientes (Gideon 2015; Glass and Fodor 2007; Mooney 2009; Ramm and Gideon 2018; Staab 2017, Van der Klein et al. 2012; Waylen 2016). El maternalismo se evidencia como una arquitectura de infraestructura pública, arreglos institucionales y legislativos construidos alrededor del supuesto de que las madres son y deben ser las cuidadoras primarias.La construcción inseparable del binomio ma- 
dre-hijo y mujer-madre fueron centrales a la incorporación de mujeres a los sistemas de bienestar en la región (Molyneux 2007) y a la formación de un tipo de ciudadanía femenina (Goldsmith 2017a), y estas ideas han tenido un efecto que permea (Gideon 2015) y ha sido resiliente al cambio (Goldsmith 2017b). Se ha documentado como concepciones maternalistas persisten en políticas de salud (Gideon 2012), están implícitas en programas de microcrédito (Martínez Franzoni and Voorend 2012) e incluso condicionan la política electoral (Franceschet, Piscopo and Thomas 2016).

Si bien la academia feminista tiende a analizar el maternalismo desde la crítica, destacando las limitaciones en los roles disponibles para mujeres y la marginación de éstas en el mercado laboral, poco se ha escrito de cómo esta construcción del cuidado también limita las posibilidades para ejercer y vivir la paternidad. Blofield and Martínez Franzoni (2015) clasifican a los países de la región según su grado de maternalismo definiendo quienes tienen un 'piso maternalista,'quienes superan esta expectativas y en cuales países se evidencia un movimiento hacia la coresponsabilidad o incluso desmadre-rización, una medida que captura el punto al cual las madres comparten porciones de responsabilidades a instituciones o al padre (Mathieu 2016). En este sentido, si bien se evidencian algunos movimientos hacia la coparentalidad en la región, cabe destacar que a diferencia de lo que se observa en países industriales avanzados (Orloff 2006), estos cambios suceden al margen de una infraestructura maternalista que se mantiene intacta. Por ejemplo, en Chile a partir del 2009 se permite transferir una parte de la licencia postnatal a los padres, lo que se puede considerar un avance en beneficios parentales.Sin embargo, el derecho a compatibilizar la paternidad con el trabajo se mantiene subyugado a la discreción y obligación maternal de organizarlo y da cuenta de un mal diseño que no promueve que los hombres entren en el territorio del cuidado. Prueba de ello es que lo toman cerca de un $0,2 \%$ de los padres, que equivale a cerca de 20 casos por mes (Lupica, 2015). En este lente de aproximación, nos proponemos un primer acercamiento a examinar la crianza en un contexto institucional maternalista desde la mirada y las experiencias de los padres.

El principal objetivo de este ‘Lente de Aproximación’ es analizar algunos discursos y experiencias de hombres latinoamericanos en torno a la paternidad. El Lente 'Proveer, cuidar y criar' hace aportes en cuanto a entender las diversas formas de experimentar y ejercer la paternidad en la región, que son variadas y están cruzadas por la edad, el ciclo vital, la clase social, la orientación sexual, la etnia, la nacionalidad, las configuraciones familiares, la situación laboral, entre otras dimensiones. La propuesta para presentar un tema monográfico para la Revista Polis está enmarcada en el Proyecto Fondecyt Regular No 1150554 (Chile) "Convertirse en Padre Hoy: Experiencias Masculinas de Transición a la Paternidad” y en el trabajo que ha realizado EME- Fundación CulturaSalud con su línea de investigación e intervención en paternidad. Buscamos observar cómo, con sus prácticas,los padres se están acercando o alejando de las normas impuestas por la masculinidad hegemónica (Connell y Messerschmidt, 2005) en sus contextos locales y globales.Los artículos incluidos están basados en investigacio- 
nes empíricas realizadas en algunos países latinoamericanos (Argentina, Brasil, Chile y México). Incluyen construcciones tanto desde paternidades liminales, como los padres encarcelados, jóvenes periféricos y experiencias de homoparentalidad, como desde arreglos tradicionales heterosexuales y en sectores socioeconómicos medios y medios altos. Estas experiencias son recolectadas y analizadas desde diversas perspectivas académicas y metodológicasutilizando métodos cualitativos, etnográficos, conocimientos del área médica y encuestas de alcance medio.

El panorama regional, tal como lo evidencian las encuestas de uso de tiempo, es que las mujeres dedican más tiempo que los hombres a tareas de cuidado incluso en familias heterosexuales de dos proveedores. Los hombres destinan 2 a 6 veces menos tiempo al cuidado de los hijos, la crianza y las tareas domésticas en el hogar (Rico y Robles, 2016). En un estudio en cuatro países se encontró que en Uruguay las mujeres destinan 33 horas por semana versus 11 horas los hombres al trabajo doméstico no remunerado. En Colombia 22 versus 7 horas, en México 39 versus 8 horas y en Perú 38 versus 14 horas (Amarante y Rossel, 2014). De este modo aún persiste una acentuada división sexual del trabajo en la región. Mientras que los hombres pasan más tiempo en el trabajo remunerado las mujeres por su parte dedican más tiempo al trabajo doméstico no remunerado. Ahora bien, las encuestas de uso de tiempo han revelado que al sumar el tiempo de trabajo remunerado más el de trabajo no remunerado en todos los países las mujeres trabajan más horas que los hombres (UNWOMEN, 2015).

En las últimas décadas la investigación en torno a la paternidad masculina ha aumentado considerablemente, tanto en el contexto euroamericano (Barclay y Lupton, 1999; Henwood y Procter, 2003; Miller, 2011) como en el latinoamericano (Aguayo y Sadler, 2011; Aguayo et al. 2011; Herrera 2013; Herrera y Pavicevic, 2016; Laguna, 2013, 2016; Olavarría, 2001a, 2001b; Valdés 2008, 2009). Algunos autoras/es han identificado un cambio a nivel ideológico en los papeles asignados a los padres (Olavarría, 2001a, 2001b; Miller 2011; Valdés 2008, 2009). Se ha hablado que las nuevas generaciones de hombres están compuestas por 'nuevos padres' que buscan distanciarse del modelo autoritario que representaba a la generación anterior. El discurso sobre el 'padre involucrado' se ha ido imponiendo en los medios de comunicación,en la cultura popular a través de imágenes en publicidad y personajes en teleseries, en los hogares y en los mismos padres. Especialmente en el caso de los padres jóvenes se espera que los hombres sean padres cercanos, cariñosos y presentes, muy diferentes al padre proveedor económico y emocionalmente distante de las generaciones anteriores (aun cuando a estos nuevos padres se les sigue exigiendo ser buenos proveedores).Pero ¿qué significa ser un padre presente?, ¿pasar un rato jugando con los/as hijos/as cada día?, ¿involucrarse activamente en algunas tareas de cuidado (comida, baños, aseo) o participar a la par con la madre en la crianza de los/as hijos/as? Madrid (2017) argumenta que los cambios en cómo se ejerce la paternidad pueden estar relacionados con una reconfiguración de la masculinidad hegemónica sin, necesariamente, producir relaciones de género más igualitarias. 
De acuerdo con Miller (2011), los supuestos esencialistas que posicionan a las mujeres como 'maternales', tengan o no hijos, siguen influenciando cómo se construye la parentalidad en los países occidentales. Los padres ocupan posiciones ambivalentes, donde se les exige mayor participación pero se les da poco espacio o se conforman con hacer unas pocas tareas de cuidado. La madre sigue siendo representada en todos los ámbitos como la cuidadora primaria ${ }^{1}$ y los padres, como cuidadores secundarios. La evidencia indica que, en la práctica, el nuevo modelo de padre involucrado es difícil de alcanzar. Las exigencias de ser un padre cercano y cariñoso se suman y superponen con las exigencias, ineludibles, de proveer y proteger a la familia en contextos de baja protección social y económica(Herrera y Pavicevic, 2016; Olavarría, 2017; Valdés y Godoy, 2008).

Estudios de transición a la paternidad realizados en distintos contextos sugieren que los hombres están interesados en participar más en la vida de sus hijos/as(Barclay y Lupton, 1999; Deave y Johnson, 2008; Draper, 2003; Finn y Henwood, 2009; Henwood y Procter, 2003; Herrera y Pavicevic, 2016; Ives, 2014; Miller, 2011; Shirani, 2015). Algunos hombres incluso se plantean disminuir su carga laboral para estar más presentes en el hogar (algo que rara vez se lleva a la práctica). Sin embargo, pocos consideran posicionarse como los cuidadores únicos o principales, especialmente si se trata de niños/as pequeños/as. Los relatos de madres y padres dan cuenta que el modelo que vincula a la mujer al cuidado y al hombre a proveer económicamente sigue vigente. Se considera que las mujeres son las que tienen los conocimientos y habilidades para cuidar y que el rol principal de los hombres es mantener económicamente a sus familias. Uno de los argumentos más usados para legitimar esta diferencia es que las mujeres tienen una disposición natural al cuidado, ya que, a diferencia de los hombres, experimentan el embarazo y la lactancia (Herrera, 2013; Herrera y Pavicevic, 2016). De esta forma, los sentimientos y conocimientos masculinos son menos válidos y legítimos en el marco reproductivo y del cuidado (McCreight, 2004; Dolan y Coe, 2011).

Existe creciente consenso respecto a que una mayor participación masculina en los cuidados y la crianza es necesaria y deseable ${ }^{2}$ (Aguayo et.al., 2017; Lupica, 2015). Además, muchos hombres anhelan ser padres presentes y activos. Sin embargo, existen obstáculos y barreras materiales (orden de género, organización del trabajo remunerado y no remunerado, discriminación salarial, falta de servicios de jardines infantiles, políticas de cuidado maternalistas, falta de políticas laborales que apoyen el cuidado) y culturales (machismo, normas de género) que dificultan el avance hacia la equidad de género en el ámbito privado y se necesitan políticas y programas para avanzar en lograr una mayor participación de los padres en la crianza.

Si bien muchos padres están dedicando más tiempo al cuidado y crianza de sus hijos todavía los cambios discursivos no se traducen en corresponsabilidad real en la distribución de tareas en los hogares (Aguayo et.al., 2017). Como se puede observar en la mayoría de los artículos inclui- 
dos en este número, hay una brecha entre el discurso del 'nuevo padre' y las prácticas familiares. Existe una enorme cantidad de familias con hijos en América Latina en las cuales el padre trae el pan a la casa y la madre está al cuidado de los hijos.El 51,6\% de las mujeres en Latinoamérica están en la casa y no trabajando remuneradamente y la principal razón es el cuidado de los hijos. En cambio, solo un 3,2\% de hombres están en esa posición (CEPAL, 2014). Muchos hombres sienten que su responsabilidad mayor es proveer económicamente y que a la madre le toca cuidar. Muchas mujeres sienten que el cuidado de los hijos/as es tarea primordial de ellas. Y las tareas están marcadas según género, como plantea Wainerman (2007).Por ejemplo en Chile los padres dedican más tiempo al juego con sus hijos que a otras tareas de cuidado (ComunidadMujer, 2018). Olavarría encontró que el discurso de igualdad de género tiene poco reflejo en la práctica (2001). Los hombres de todas las edades y condición social dicen involucrarse en alguna medida en la crianza de sus hijos/as. Sin embargo, ven las tareas asociadas a la auto-reproducción de la familia como ajenas, con la excepción de aquellas actividades que refuerzan la masculinidad dominante (arreglos de la casa, trámites, presupuesto). Muchos hombres ven su participación en lo doméstico como una ‘colaboración’ o ‘ayuda’ a la mujer (generalmente por período acotados). La discriminación salarial a las mujeres y la precariedad laboral refuerzan este orden manteniendo a los hombres en el trabajo y a las mujeres en el hogar (ComunidadMujer, 2018). Por otra parte, tradicionalmente las políticas de familia han sido de corte maternalista responsabilizando a las madres del cuidado de los hijos/as (Blofield y Martínez, 2014).Los padres han sido incluidos sólo recientemente y de forma tímida. Las resistencias institucionales y culturales hacia mayor equidad de género en el ámbito del cuidado todavía son fuertes (Blofield y Martínez, 2014; Olavarría, 2017).

A pesar de ello se pueden ver algunos avances, aunque lentos, hacia la corresponsabilidad, especialmente en familias en las cuales tanto padre como madre trabajan remuneradamente, es decir, familias con al menos dos proveedores económicos. No obstante, las mujeres, incluso en esta forma familiar, continúan dedicando más tiempo a la crianza y a lo doméstico que los hombres (Aguayo, Correa y Cristi, 2011). También es importante considerar que existen diferentes arreglos familiares, cada vezmás frecuentes y visibles, donde el padre está a cargo de sus hijos/as. Hay parejas heterosexuales que desafían al orden tradicional de género en las cuales el padre es el cuidador principal y la madre trae el pan a la casa. Las parejas de hombres criando hijas/os también desafían la noción de que la cuidadora primaria debe ser una mujer (Laguna y Herrera et al. en este número). Finalmente, cabe preguntarse si la asociación del cuidado y lo femenino no ha llevado a invisibilizar las experiencias masculinas de cuidado (Gonzálvez, 2016). En este Lente de Aproximación se profundiza sobre algunos de los temas expuestos.

Los artículos de Armijo y Saldaña presentan resultados de investigaciones que analizan las experiencias de paternidad de hombres en parejas heterosexuales en Chile.Ambos artículos se preguntan por la equidad de género al interior de estas familias. 
El artículo "Los discursos de legitimación de los padres trabajadores chilenos sobre la reproducción del cuidado” de Lorena Armijo aborda el tema de la participación de los padres en el cuidado de los hijos desde el marco teórico de la reproducción social de Pierre Bourdieu. Los hallazgos presentados se basan en una investigación empírica realizada en Santiago de Chile que consistió en 29 entrevistas semiestructuradas y 8 grupos de discusión a padres trabajadores heterosexuales con pareja e hijos menores de edad. Estos hombres ven la paternidad como un proyecto en construcción donde equivocarse y aprender de los errores está permitido. El convertirse en padres implica dejar atrás una vida centrada en sí mismos para elegir criar y cuidar. Ser padre se distingue de ser madre con argumentos biologicistas relacionados con el apego y el cuerpo femenino. Los padres que eligen cuidar concilian trabajo y cuidado en sus propios términos, dedicándole menos tiempo al cuidado que las mujeres y construyendo una autoimagen de heroicidad. En esta materia, los padres no se comparan con las madres, ya que ellas no son sus iguales.Los 'buenos padres' cuidan desde su masculinidad de manera complementaria a la 'buena madre'. Armijo argumenta que no existe una subversión del orden de género y que los cambios discursivos no han conllevado cambios sustantivos en las prácticas y significados asociados a la paternidad.

El artículo "Relaciones de género y arreglos domésticos: masculinidades cambiantes en Concepción, Chile” aborda la organización del trabajo doméstico y parentalidad en el contexto de la creciente participación femenina en el mercado laboral en la ciudad de Concepción, Chile.A través de entrevistas grupales, individuales y grupos de discusión con parejas heterosexuales con hijas e hijos menores de 14 años, Saldaña da cuenta de una heterogeneidad en los patrones y arreglos familiares.Esta investigación ocurre en el contexto de un país Latinoamericano (Chile) con bajos niveles regionales de inserción laboral femenina donde las mujeres destinan más del doble de horas semanales a trabajos no remunerados con respecto a sus pares masculinos y un estado maternalista que amarra beneficios para menores y su crianza a mujeres y concluye con un llamado a políticas públicas que buscan conciliar tareas laborales y familiares.Dentro de sus hallazgos, da cuenta de un liderazgo femenino en la organización doméstica refiriéndose a que las mujeres guían a sus parejas en aquellas labores y responsabilidades que son compartidas, quienes mantienen en áreas de privilegio masculino constituidos por la discrecionalidad 'para decidir cuándo y qué labores realizar'.Más allá de la familia, las redes de apoyo de cuidado se mantienen fuertemente feminizadas.La autora observa un proceso gradual de cuestionamiento de los roles de género y una búsqueda de complementariedad y se evidencian algunos casos de rupturas de relaciones tradicionales de género en parejas más jóvenes.Si bien, en su artículo se observa una persistencia de roles de género tradicionales y asimétricos, el mayor involucramiento cotidiano y la cercanía afectiva es medular a la configuración identitaria de los padres entrevistados.

Los trabajos de Fiterman y Vaz de Campos Moreira y de Morales, Catalán y Pérez nos describen las experiencias de los hombres en la primera 
etapa de su paternidad. Ambos textos exponen las dificultades, contradicciones y tensiones que estos padres enfrentan. Todavía se observa que muchos hombres se ven en un rol secundario en comparación con la madre, como ayudantes o telespectadores.

En el artículo "O pai na gestação, no parto e aos três meses de vida do primeiro filho” de Hannah Fiterman y Lúcia Vaz de Campos Moreira se investiga la participación de los padres durante la gestación, parto y en los primeros meses de vida, etapas fundamentales en el comienzo del ejercicio de la paternidad. Se realizó un estudio cualitativo con 30 padres primerizos de clases media en Salvador/Bahia, Brasil. La paternidad en estas etapas está marcada por transiciones o cambios importantes, por procesos de reflexión acerca de sus modelos de paternidad y de cómo quieren ser padres, también acerca de sus responsabilidades económicas. En la gestación la mayoría de los padres se consideran en un proceso de transición, entre no sentirse padres y sentirse como tales. Algunos se sienten padres embarazados (pai gravido) mientras que otros viven el proceso con mayor distancia y se perciben como futuros padres o teleespectadores del proceso. La participación en procesos como el parto refuerza el involucramiento con el hijo/a y el apoyo a la madre. En los primeros meses los padres se consideran en general satisfechos con la experiencia sin embargo ven el trabajo como una gran limitación para estar más presentes. Es una etapa de aprendizaje a partir de la experiencia y de observar a la madre como cuida y de compartir experiencias con otros padres y madres. Algunos padres viven estos procesos con ansiedades y tensiones.

El artículo "Los padres también se deprimen en el postparto: comprendiendo el fenómeno desde la voz de sus protagonistas" trae a las ciencias sociales un tema que si bien es muy común -se presenta en uno de cada diez padres-, ha tenido poco espacios en las discusiones sobre parentalidad: el de la depresión postnatal masculina.Morales, Catalán y Pérez aportan a la discusión con un primer análisis exploratorio del caso chileno.Las autoras exploran el significado y sentido de la depresión postnatal masculina a través de entrevistas en profundidad a padres con alto puntaje en una escala usada para diagnósticos preliminares de depresión postnatal. Ocupan categorías analíticas relacionadas tanto a contenidos como procedimentales y encuentran que un factor común es la situación de un embarazo no planificado donde la llegada del bebé es experimentada como una 'irrupción en la vida'.Al igual que en otros artículos que componen este lente de aproximación, los entrevistados expresan una visión tradicional de su rol, como proveedor económico principalmente; y solamente secundario en relación a proveer cuidados.A pesar de las diferencias en estatus con los jóvenes en barrios populares analizados por Hasisic (ver más adelante) - hablamos aquí de mayores de edad con empleos formales y niveles medios de educación formalellos también expresan inseguridades al asumir las tareas de cuidado.De esta forma, en este análisis se visualiza una vulnerabilidad que aparece como rupturista o que al menos comienza a desarticular construcciones patrimoniales de masculinidad y a su vez se releva la paternidad como 
un proceso que puede necesitar apoyos desde un punto de la salud mental.

Los estudios de Hasicic y de Garay et al.Indagan en la paternidad de hombres jóvenes, pero en distintas situaciones vitales (jóvenes populares en Argentina y jóvenes privados de libertad en Brasil). En los dos casos, los jóvenes buscan un lugar desde el cual ejercer su paternidad y se preguntan qué es ser un 'buen padre'. Estos jóvenes tampoco cuestionanque la madre sea considerada la cuidadora principal.

En el artículo “Jóvenes y cuidado: un análisis sobre las prácticas de crianza y cuidado de padres varones de un barrio popular del Gran La Plata (Argentina)”, Cintia Hasicic indaga en la distribución de tareas de cuidado entre padres jóvenes en barrios populares de la capital Argentina. A través de un levantamiento empírico original, hace aportes conceptuales innovadores sobre la categorización y contenidos de las relaciones de cuidado parental.Ocupando técnicas de reclutamiento vía bola de nieve, encuentra un mayor involucramiento con respecto a sus propios padres consistente con el mayor involucramiento observado en la literatura sobre otros países y contextos.Sus prácticas se diferencian de sus experiencias con sus propios padres que incluyen ausencias, abandono o violencia y logran superar una sensación inicial de no encontrar su lugar en el cuidado (ver Morales et al. en este número).La autora procede a desempacar las tareas de cuidado encontrando construcciones de cuidado diferenciados para hijas e hijos. Concluye que si bien las relaciones de cuidado obedecen principalmente al concepto de ayuda (como menciona Saldaña en este número), también incluyen lo que Hasicic define como 'cuidados periféricos' referidos a actividades lúdicas o administrativas.

En el artículo "Sentidos e práticas de paternidade: vozes de homens jovens em privação de liberdade”, Jimena de Garay Hernández, Anna Paula Uziel, Marcos Antônio Ferreira do Nascimento y Gabriela Salomão Alves Pinho indagan en la producción de masculinidades en hombres jóvenes privados de libertad. Es un tema relevante porque son padres que no residen con sus hijos en razón de que están privados de libertad, por el derecho de los hijas/os a ver a sus padres, porque el papel de la institución y de las madres en facilitar o no el acceso a los hijos es crucial y porque hay evidencia que plantea que la paternidad es una dimensión que puede ayudar a salir de la cultura del delito o del narcotráfico. Las instituciones de privación de libertad no promueven la paternidad de los hombres de igual manera que la maternidad en el caso de mujeres infractoras. Se realizó un estudio cualitativo con diferentes actores de unidades en las cuales los infractores cumplen sus medidas de internación. Muchos de los jóvenes no tuvieron un padre presente. Varios tienen una visión restringida de la paternidad, como llevar a la plaza y comprar cosas. La paternidad se instala discursivamente en el horizonte de ser un buen ejemplo para los hijos, ser un hombre de bien. Los entrevistados se ven como proveedores - de sus madres y de sus hijos- a pesar de las limitaciones del contexto en el que se encuentran y ven las actividades ilícitas como el trabajo que les permitía cumplir con ese papel. 
La paternidad es vista como una performance de la masculinidad potente y es vivida con ostentación. Al mismo tiempo es una experiencia frágil ya que una mala relación con la madre de su hijo/a o si ella tiene otra pareja puede hacer que no traiga más a su hija/o a visitas. Por otra parte, en el grupo estudiado, se observaron varios desafíos para la salud sexual. Varios entrevistados no usaron preservativo intencionalmente para sentir más placer o tener varias parejas sexuales.

Los trabajos de Herrera et al. y de Laguna abordan las experiencias de padres gay en Chile y México. Los dos artículos tienen varios puntos en común, como por ejemplo, la dificultad de compatibilizar dos identidades que parecen excluyentes (gay y padre), los efectos de la discriminación y la homofobia en estas familias y cómo, mediante la asimilación,buscan alcanzar la validación social.

En el artículo “'Soy un papá súper normal’: Experiencias parentales de hombres gay en Chile," se abre una ventana hacia experiencias de homoparentalidad en Chile.A través de entrevistas en profundidad analizadas con categorías teóricas a-priori y emergentes, encuentran diferencias entre quienes fueron padres en el marco de una relación heterosexual anterior y quienes buscaron ser padres en el contexto de una relación homosexual, y que deben desplegar una cantidad considerable de recursos económicos y energéticos para este fin. La principal conclusión de Herrera, Miranda, Pavicevic y Sciaraffia es que más como categorías mutualmente exclusivas o incompatibles, la homosexualidad y el deseo y/o experiencia de ser padres coexisten. Concluyen que si bien la mayoría de los desafíos son compartidos con padres heterosexuales, las familias homoparentales se encuentran con la necesidad de desarrollar estrategias de protección para sus familias en un contexto adverso: heteronormativo y frecuentemente homofóbico.Las/el autor/as encuentran que las familias con mayores recursos económicos pueden desarrollar mejores estrategias a la hora de escoger y crear un entorno seguro y muchas de estas buscan la asimilación, reproduciendo modelos de familias nucleares, biparentales.En el contexto de este lente de aproximación, este artículo hace un aporte particularmente innovador desafiando tanto los estereotipos sobre la homosexualidad como aquellas patriarcales donde se asume una mujer como imprescindible para la crianza.

En el artículo "Paternidad de hombres gay: ¿Los albores de una neoparentalidad?”, el autor nos presenta una encuesta aplicada a jóvenes gay y entrevistas en profundidad a padres gay que son analizadas con enfoque crítico de género y desde el interaccionismo simbólico.Encuentra que quienes deciden tener hijos deben sortear barreras biológicas, restricciones legales, y lo que aparece aquí como lo más difícil de sobrellevar: los constructos culturales homofóbicos. Tanto así que en algunos jóvenes encuestados se reproduce la noción de incompatibilidad entre su orientación sexual y una futura paternidad.Al igual que Herrera et al., encuentra que las familias homoparentales buscan apegar sus prácticas y la conformación de la familia a 'normas socialmente validadas.'Sin embargo, sus 
entrevistados encuentran dificultades a la hora de construir parentalidad desde el espacios liminal de la homosexualidad y de esta forma terminan por construir innovaciones.El autor nos cuenta de las prácticas de estas familias que incluyen una necesidad frecuente de demostrar su capacidad como padre, una des-generación de las tareas de cuidado; la creación de 'fachadas' de familias convencionales en los cuales las y los niñas/os son partícipes de guardar el 'secreto'; el encontrar apoyo en redes no familiares; y el intento de entregar una educación sobre género consciente y paulatina.

Este Lente de Aproximación busca aportar al debate en este campo emergente de la paternidad y el cuidado, ofreciendo algunas evidencias, reflexiones y distinciones desde América Latina. Queda un largo camino por recorrer para avanzar hacia la corresponsabilidad y equidad de género en el cuidado entre hombres y mujeres. En la región aúnse mantienen enfoques que sobrerresponsabilizan a las madres del cuidado y ubican a los padres en la proveeduría económica.

Consideramos que algunos de los desafíos pendientes en el tema de paternidad son:

- Cuestionar el paradigma de que es la madre quien tiene que cuidar y el padre quien tiene que proveer. Se necesita lograr la voluntad política de los países para avanzar en corresponsabilidad y en igualdad de género en el campo del cuidado y la crianza de las/los hijas/os y en las tareas domésticas. Para avanzar en este sentido se debe ampliar la mirada desde políticas maternalistas que responsabilizan exclusivamente a la mujer del cuidado de los/as hijos/as hacia políticas de cuidado diseñadas con equidad de género.

- La legislación de familia tampoco debe asumir que la madre es la principal cuidadora. En casos de separación o no residencia con los hijos/as, los padres deben tener condiciones para acordar la custodia compartida o acercarse a ella a través de cuidados personales flexibles (y no un régimen mínimo de contacto con sus hijos para los padres). Esta es una necesidad urgente en parejas de dos proveedores.

- Crear postnatales masculinos largos, además de buenos postnatales para las madres. Los mejores diseños son de un tiempo exclusivo para el padre, intransferible -que se ha llamado cuota de paternidady cubierto por el estado. Esta es una política maestra para lograr que los padres se involucren de verdad en el cuidado de sus hijas/os y para avanzar en la igualdad de género en el cuidado. Los postnatales masculinos en la región otorgan de dos a catorce días a los padres (usualmente solo para aquellos con contrato) (Aguayo et.al., 2017). Esto está muy lejos de la experiencia de países más igualitarios en género, como Suecia, Islandia o Noruega.

- Diseñar programas de paternidad que permitan llegar a los padres en sus comunidades e instituciones instalando mayor conciencia de 
la importancia de su participación en el cuidado, así como cuestionado las normas de género y el machismo, que son barreras culturales para el avance en la corresponsabilidad.

- Las políticas y programas de paternidad no deben usarse para promover la familia heterosexual como modelo social. Es importante que las familias diversas tengan reconocimiento y que sus derechos sean resguardados.

- No se debe promover solo la paternidad biológica como única posibilidad legítima de paternidad. Muchos niños/as son cuidados por padres adoptivos, abuelos, tíos, padrastros o padres de la diversidad sexual.

- Las tareas de cuidado deben tener mayor valoración y reconocimiento social. 
Polis, Revista Latinoamericana, $N^{\circ}$ 50, 2018

\section{Notas}

${ }^{1}$ De acuerdo con la encuesta CASEN 2011 en Chile los padres son señalados como cuidadores principales sólo en un 4\% (y 15\% como cuidadores secundarios).

${ }^{2}$ Aunque también hay reservas frente al proyecto de involucrar a los hombres. En aquellos casos en los que los padres han estado ausentes, o en aquellos en que ejercen violencia contra la pareja y/o contra los hijos/as. Además se debe tomar en cuenta que la figura del padre no existe en todas las configuraciones familiares (algunos ejemplos son las familias construidas por madres solteras por elección o por dos madres). 


\section{Bibliografía}

Aguayo, F. y M. Sadler (2011)El papel de los hombres en la equidad de género:¿qué masculinidades estamos construyendo en las políticas públicas en Chile?. En Masculinidades y Políticas Públicas: Involucrando Hombres en la Equidad de Género, editado por Francisco Aguayo y Michelle Sadler, 105-126. Santiago, Chile: Universidad de Chile.

Aguayo, F., P. Correa y P. Cristi (2011)Encuesta IMAGES Chile: Resultados de la encuesta internacional de masculinidades y equidad de género.Santiago, Chile: CulturaSalud/EME.

Aguayo, F., Levtov, R., Barker, G., Brown, V. y Barindelli, F. (2017).Estado de la paternidad: América Latina y el Caribe 2017. Nueva York: IPPF/ RHO, Washington, D.C., EUA: Promundo-US

Amarante, V. y Rossel, C. (2014). Unpaid Household Work in Latin America: Unfolding Patterns and Determinants. 33rd IARIW General Conference. Rotterdam, Paises Bajos: International Association for Research in Income and Wealth.

Barclay, L., \& Lupton, D. (1999). The experiences of new fatherhood: A socio-cultural analysis. Journal of Advanced Nursing, 29(4), 10131020. doi: 10.1046/j.1365-2648.1999.00978.x

Blofield, M. and Martínez, J. (2014). Trabajo, familia y cambios en la política pública en América Latina: Equidad, maternalismo y corresponsabilidad. Revista de la Cepal 114, 108-125.

Blofield, M., y J. Martinez Franzoni (2015)Maternalism, Co-responsability and Social Equity: A Typology of Work-Family Policies.Social Politics: International Studies in Gender, State and Society 22 (1):38-59.

CEPAL (2014). La medición del tiempo y el aporte de las mujeres a la economía. Notas para laigualdad N¹5.

ComnidadMujer (2018) Mujer y trabajo: Cuidado y nuevas desigualdades de género en la división sexual del trabajo. Santiago, Chile: Comunidad Mujer.

Connell, R. W., y Messerschmidt, J. W. (2005). HEGEMONIC MASCULINITY: Rethinking the Concept. Gender \& Society, 19(6), 829-859.

Dolan, A., y Coe, C. (2011). Men, masculine identities and childbirth. Sociology of Health \& Illness, 33(7), 1019-1034. 
Franceschet, S., J. Piscopo M. y G. Thomas. (2016). Supermadres, Maternal Legacies and Women's Political Participation in Contemporary Latin America. Journal of Latin American Studies 48 (1):1-32.

Gideon, J. (2012). Engendering the Health Agenda? Reflections on the Chilean Case, 2000-2010. Social Politics 19 (3):333-360.

Gideon, J. (2015).Gender, Globalization, and Health in a Latin American Context. NY, EUA: Palgrave Macmillan.

Glass, C., y E. Fodor.(2007). From Public to Private Maternalism? Gender and Welfare in Poland and Hungary after 1989.Social Politics 14 (3):323-50.

Goldsmith Weil, J. (2017a). Milk Makes State: The Extension and Implementation of Chile's State Milk Programs, 19017-1971.Historia 50 (I).

Goldsmith Weil, J. (2017b). Using Critical Junctures to Explain Continuity: The Case of State Milk in Neoliberal Chile. Bulletin of Latin American Research 36 (1):52-67.

Henwood, K., y Procter, J. (2003). The 'good father': Reading men's accounts of paternal involvement during the transition to first-time fatherhood. British Journal of Social Psychology, 42(3), 337-355. doi: 10.1348/014466603322438198

Herrera, F. (2013). Men always adopt: Infertility and reproduction from a male perspective. Journal of Family Issues, 34(8), 1059-1080. doi: 10.1177/0192513X13484278

Herrera, F., y Pavicevic, Y. (2016). Anticipando la paternidad: ‘ella es la que está embarazada’. Masculinities and Social Change, 5(2), 107-133. doi: 10.17583/MCS.2016.2038

Ives, J. (2014). Men, maternity and moral residue: Negotiating the moral demands of the transition to first time fatherhood. Sociology of Health and Illness, 36(7), 1003-1019. doi: 10.1111/1467-9566.12138.

Lupica, C. (2015). Corresponsabilidad de los cuidados y autonomía económica de las mujeres: Lecciones aprendidas del Permiso Postnatal Parental en Chile. Santiago, Chile: CEPAL, Serie Asuntos de Género. Naciones Unidas.

Madrid, S. (2017). The good night kiss: fatherhood among corporate managers and the reconfiguration of hegemonic masculinity in Chile, en International Journal for Masculinity Studies. Vol. 12, Issue 3-4.

Martínez Franzoni, J., y K. Voorend. (2012). Blacks, Whites, or Grays? Conditional Transfers and Gender Equality in Latin America.Social Politics 19 (3):383-407. 
Mathieu, S. (2016). From the Defamilialization to the 'Demotherization' of Care Work.Social Politics 23 4): 576-91.

Van der Klein, M; R. Plant, N. Sanders and L. R Weintrob (eds). (2012). Maternalism Reconsidered: Motherhood, Welfare and Social Policy in the Twentieth Century, New York- Oxford, EUA: Berghahn Books.

Waylen, G. ed. (2016).Gender, Institutions, and Change in Bachelet's Chile, Studies of the Americas. New York, EUA: Palgrave Macmillan.

McCreight, B. S. (2004). A grief ignored: narratives of pregnancy loss from a male perspective. Sociology of Health \& Illness, 26(3), 326-350.

Miller, T. (2011). Making sense of fatherhood: Gender, caring and work. Cambridge,UK: Cambridge University Press.

Molyneux, M. (2007).Change and Continuity in Social Protection in Latin America,Mothers at the Service of the State? : United Nations Research Institute for Social Development.

Mooney, J. y E. Pieper. (2009).The Politics of Motherhood: Maternity and Women's Rights in 20th Century Chile. Pittsburh, PA: University of Pittsburgh Press.

Olavarría, J. (2001a). ¿Hombres a la deriva?: poder, trabajo y sexo. Santiago, Chile: Flacso-Chile.

Olavarría, J. (2001b). Y todos querían ser (buenos) padres: Varones de Santiago de Chile en conflicto. Santiago, Chile: Flacso-Chile.

Olavarría, J. (2017) Sobre hombres y masculinidades. “ponerse los pantalones".Santiago, Chile: UAHC.

Orloff, A. S. (2006b)From Maternalism to Employment for All': State Policies to Promote Women's Employment across the Affluent Democracies. In State After Statism: New State Activities in the Age of Liberalization, 231-68. Cambridge and London: Harvard University Press.

Ramm, A. y J. Gideon (eds).(Manuscrito bajo revision).Unpacking Maternalism.

Staab, S. (2012)Maternalism, Male-Breadwinner Bias, and Market Reform: Historical Legacies and Current Reforms in Chilean Social Policy.Social Politics: International Studies in Gender, State and Society 19 (3):299332.

Staab, S. (2017)Gender and the Politics of Gradual Change: Social Policy Reform and Innovation in Chile. New York, EUA: Palgrave Macmillan. 
UNWOMEN (2015). Progress of the World's Women 2015-2016: Transforming Economies, Realizing Rights.New York, EUA: UNWOMEN

Valdés S, X. (2009). El lugar que habita el padre en Chile contemporáneo: Estudio de las representaciones sobre la paternidad en distintos grupos sociales. Polis (Santiago), 8(23), 385-410.

Valdés, X. y Godoy, C.G. (2008). El lugar del padre: rupturas y herencias. Representaciones de la paternidad en grupos altos, medios y populares chilenos. Estudios Avanzados 6(9), 79-112.

Wainerman, C. (2007). Conyugalidad y Paternidad ¿Una revolución estancada? En Gutiérrez, M.A (Ed.). Género, familias y trabajo: Rupturas y continuidades. Desafíos para la investigación política (pp. 179222). Buenos Aires, Argentina: CLACSO 